\title{
Unraveling proteome changes and potential regulatory proteins of bovine follicular Granulosa cells by mass spectrometry and multi-omics analysis
}

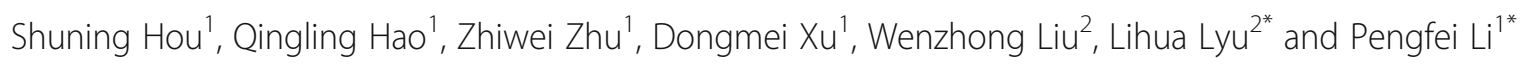

\begin{abstract}
Background: In previous study, we performed next-gene sequencing to investigate the differentially expressed transcripts of bovine follicular granulosa cells (GCS) at dominant follicle (DF) and subordinate follicle (SF) stages during first follicular wave. Present study is designed to further identify the key regulatory proteins and signaling pathways associated with follicular development using label-free liquid chromatography-tandem mass spectrometry (LC-MS/MS) and multi-omics data analysis approach.

Methods: DF and SF from three cattle were collected by daily ultrasonography. The GCs were isolated from each follicle, total proteins were digested by trypsin, and then proteomic analyzed via LC-MS/MS, respectively. Proteins identified were retrieved from Uniprot-COW fasta database, and differentially expressed proteins were used to functional enrichment and KEGG pathway analysis. Proteome data and transcriptome data obtained from previous studies were integrated.

Results: Total 3409 proteins were identified from 30,321 peptides (FDR $\leq 0.01$ ) obtained from LC-MS/MS analysis and 259 of them were found to be differentially expressed at different stage of follicular development (fold Change $>2, P<0.05)$. KEGG pathway analysis of proteome data revealed important signaling pathways associated with follicular development, multi-omics data analysis results showed 13 proteins were identified as being differentially expressed in DF versus SF.

Conclusions: This study represents the first investigation of transcriptome and proteome of bovine follicles and offers essential information for future investigation of DF and SF in cattle. It also will enrich the theory of animal follicular development.
\end{abstract}

Keywords: Bovine, Follicle, Proteomic analysis, Multi-omics data analysis, Label-free

\section{Introduction}

Mono-ovulatory species such as cattle undergoes 2-3 follicular wave during one estrous/menstrual cycle. In each follicle wave, a group of follicles begin to grow and only one of them get selected to become DF and cause regression of remaining group of follicles known as SF [1]. In general, only the DF eventually ovulates, the meiotically

\footnotetext{
*Correspondence: lihualvsxau@126.com; adamlpf@126.com

${ }^{2}$ College of Animal Science and Technology, Shanxi Agricultural University, Taigu 030801, Shanxi, China

${ }^{1}$ College of Life Science, Shanxi Agricultural University, Taigu 030801, Shanxi, China
}

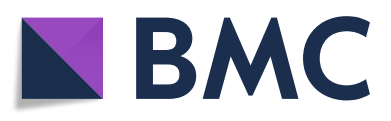

(๑) The Author(s). 2019 Open Access This article is distributed under the terms of the Creative Commons Attribution 4.0 International License (http://creativecommons.org/licenses/by/4.0/), which permits unrestricted use, distribution, and reproduction in any medium, provided you give appropriate credit to the original author(s) and the source, provide a link to the Creative Commons license, and indicate if changes were made. The Creative Commons Public Domain Dedication waiver (http://creativecommons.org/publicdomain/zero/1.0/) applies to the data made available in this article, unless otherwise stated.

competent oocyte and transform into progesterone secreting CL gland. Both of these events are critically important as the quality of ovulated oocyte determines the success of early embryo development and secretory CL progesterone establish and maintain the pregnancy in cattle. Previous research indicated that follicular development is closely associated with the estrous behavior, estrous cycle, oocyte competency and embryo survival rate [2]. However, any abnormality or reproductive disorders associated with follicular development can be a major cause of infertility in cow as well as human [3]. Therefore, it is critical to understand the molecular mechanism regulating normal 
follicular development in cattle. Several studies have been performed in the past to understand the physiological regulation of follicular development modulation and ovulation mechanism, such as gonadotrophin interacts with follicular growth factor [4-6], the relationship between apoptosis of follicle GCs and follicle atresia [7-10], the influence of hormone such as estrogen and progesterone [11], the role of growth factor such as vascular endothelial growth factor (VEGF) and insulin-like growth factors 1 (IGF-1) [12-14], the effect of concentration of reactive oxygen [15]. In addition, transcriptome of follicles at different developmental stage have also been analyzed to understand the molecular mechanism regulating bovine follicular development [16]. However, characterizing the functionally available proteins resulting from mRNA translation would provide novel insight into the signaling pathways and biomarkers of follicular developmental process in cattle. As per our knowledge, this is the first study where we characterized the proteome of bovine GCs at DF and SF in first follicular wave and performed an integrated analysis of protein abundance and transcript level to reveal the molecular regulation of bovine follicular development.

\section{Materials and methods}

\section{Animal care}

All animal procedures were implemented in strict accordance with the principles outlines in "Guide for the care and use of Laboratory Animals" by National Institutes of Health.

\section{Samples collection}

Three Holstein dairy cows selected for estrus synchronization with prostaglandin F2 $\alpha$ (PGF2 $\alpha$ ), follicular diameter and growth were observed and recorded by daily ultrasonography, ovaries were removed from cows when the largest follicle appeared and the growth rate was significantly higher than growth rates of other follicles, and the diameter of largest follicle is $8.5 \mathrm{~mm}$, the largest follicle and second largest follicle were collected and considered as DF and SF, respectively. GCs from DF and SF were isolated and stored at $-80^{\circ} \mathrm{C}$ until used for further experiment.

\section{Extraction and digestion of total proteins}

GCs were lysed by ultrasonication and homogenization in cold extraction buffer (7 M urea, $40 \mathrm{mM}$ Tris- $\mathrm{HCl}, 1 \%$ dithiothreitol, $1 \mathrm{mM}$ EDTA, $1 \%$ Protease Arrest). The lysate was centrifuged at $4{ }^{\circ} \mathrm{C}$ for $40 \mathrm{~min}$ and supernatants were collected and filtered for protein quantification using Bradford Coomassie ${ }^{\bullet}$ Brilliant Blue G-250 method. After quantification, $50 \mu \mathrm{g}$ of protein from each sample were denatured using $1 \mathrm{M}$ urea and incubated for $10 \mathrm{~min}$ at room temperature, followed by addition of $10 \mathrm{mM}$ dithiothreitol and incubation for $1 \mathrm{~h}$ at $56^{\circ} \mathrm{C}$. Proteins were then alkylated with $55 \mathrm{mM}$ iodoacetamide in the dark for $40 \mathrm{~min}$ at room temperature and were further subjected to digestion with $1 \mu \mathrm{g}$ trypsin for 16 $17 \mathrm{~h}$ at $37^{\circ} \mathrm{C}$ [17].

\section{Label-free liquid chromatography-tandem mass spectrometry (LC-MS/MS)}

The content of each peptide was determined and quantified by Capillary High Performance Liquid Chromatography (Eksigent 425, AB SCIEX) and label-free mass spectrometry [18]. (A) $\mathrm{H}_{2} \mathrm{O}+0.1 \%$ formic acid and (B) acetonitrile $+0.1 \%$ formic acid, were consisted of the mobile phase. Desalting of the samples was performed online using a reversed-phase C18 trapping column (0.1 $\mathrm{mm}$ internal diameter, $20 \mathrm{~mm}$ length, $3 \mu \mathrm{m}$ particle size; Waters). The peptides were then separated using nanocolumn $(0.75 \mathrm{~mm}$ internal diameter, $150 \mathrm{~mm}$ length, $5 \mu \mathrm{m}$ particle size; Waters) at $0.3 \mu \mathrm{L} / \mathrm{min}$. Peptides were eluted from the column using the following gradient: 5$80 \% \mathrm{~B}$ in $110 \mathrm{~min}, 80-5 \% \mathrm{~B}$ in $0.1 \mathrm{~min}$, maintained at $5 \%$ for $120 \mathrm{~min}$ and then back to initial conditions. Mass spectrometer (Q-Exactive; Thermo Scientific, USA) was connected to the liquid chromatography apparatus to detect the eluted peptides. The separated peptide fragments were identified by mass spectrometer operated in positive ion mode with electrospray ionization and collision-induced dissociation (CID). Full-scan MS spectra $(350-1750 \mathrm{~m} / \mathrm{z})$ was acquired at a resolution of 70 , 000 with an automatic gain control (AGC) target value of $3 \mathrm{e} 6$ by electrospray ionization. The full-scan maximum injection time was $20 \mathrm{~ms}$ (millisecond), and the dynamic exclusion was set to $25.0 \mathrm{~s}$. CID spectra were acquired at a resolution of 17,500 with an AGC target value of $2 \mathrm{e} 5$ and a maximum injection time of $80 \mathrm{~ms}$. The isolation window was set to $2.0 \mathrm{~m} / \mathrm{z}$.

\section{Data analysis}

Raw data were imported into Expressionists software (Proteome Discover 2.0) for processing [19], the quantification was performed based on the peak intensities of the report ions of the only unique peptides in the MS/ MS Spectra. MS/MS spectra were searched against the Uniprot-COW FASTA database (version 2017-10) with the following mascot parameters [20, 21]: peptide mass tolerance for $\pm 15 \mathrm{ppm}$ and fragment mass tolerance for $20 \mathrm{mmu}$. Trypsin was used as the protein-cleaving enzyme, and the two missed cleavages were accepted. Carbamidomethylation of cysteine was designated as a fixed modification, and oxidation of methionine, acetylation on protein $\mathrm{N}$-term were selected as variable modifications. The peptide confidence was high, and the peptide length was set to $>4$. The peptide false discovery rate (FDR) was set to $\leq 0.01[22]$. 


\section{Bioinformatics analysis}

Gene ontology (GO) assignments (http://www.geneontology.org) were used to classify the functions of the differentially expressed proteins (fold change $\geq 2$ ), and could be categorized into three main categories: biological process, cellular component and molecular function. The most important biochemical metabolic pathways and signal transduction pathways were identified by PANTHER and KEGG PATHWAY (http://www. genome.jp). Cluster of Orthologous Groups (COG) of proteins analysis was used to cluster the original functions of proteins (http://www.pantherdb.org).

\section{Multi-omics data analysis}

Venn diagram and heatmap were used as tools for multi-omics correlation analysis. Overlap of differentially expressed genes between transcriptome and proteome was displayed by venn diagram. As an intuitive figure, a heatmap could visualize the matrix data by representing individual values with different colours.

\section{Western blotting for validation}

We have validated three proteins (OGN, ROR2, HSPB1) and experiment details are available on 《Proteomic characterization of bovine granulosa cells in dominant and subordinate follicles》 [23].

\section{Results}

Top 30 highly differentially expressed proteins in DF and SF

The top 30 highly differentially expressed proteins in GCs of bovine DF and SF are shown (Table 1), including all 26 upregulated proteins and 4 downregulated proteins.

\section{Go analysis}

One of the main uses of the GO is to perform enrichment analysis on protein sets. Total 3409 proteins were identified from the 30,321 peptides sequences obtained from LC-MS/MS (FDR $\leq 0.01$ ) (Additional file 1: Table S1). Out of 3409, 259 were differentially expressed including 26 upregulated proteins (fold change $\geq 2$ ) and 233 downregulated proteins (fold change $\leq 0.5$ ) in DF (Additional file 2: Table S2). The differentially expressed proteins were categorized under 3 major GO classifications: biological process, cellular component and molecular function (Additional file 3: Table S3). Among the biological processes, many biological processes were associated with follicular development such as cellular process $(17.55 \%)$, metabolic processes (14.78\%), regulation of biosynthetic process $(9.31 \%)$ and cell death (1.55\%) (Fig. 1). With respect to their molecular function, most of the proteins were involved in nucleic acid binding (20.04\%) and protein binding (17.89\%) (Fig. 2). Regarding cellular components, most of the proteins were assigned to the nucleus (11.46\%) and cytoplasm (18.69\%) (Fig. 3).

\section{KEGG pathway analysis}

KEGG pathway analysis results showed the most important biochemical metabolic pathways and signal transduction pathways were associated with follicular development (Additional file 4: Table S4). such as estrogen signaling pathway, oocyte meiosis, progesterone-mediated oocyte maturation, apoptosis signaling pathway, insulin signaling pathway and p53 signaling pathway, etc. Especially, PI3K-Akt signaling pathway is classical signaling pathway in follicular development regulation, including 8 differentially expressed proteins in PI3K-Akt signaling pathway (HSP90AB1, GNG10, YWHAG, YWHAH, YWHAB, YWHAQ, RPS6, CDC37) (Additional file 5: Figure S1). Also, HSP90AB1 is involved in estrogen signaling pathway and progesterone-mediated oocyte maturation, RPS6 involved in mTOR signaling pathway.

\section{COG analysis}

PANTHER was used to categorize differentially expressed proteins as different functional contents, and annotated set of 215 bovine genes as a reference dataset. 187 differentially expressed proteins were categorized for 19 functional contents through COG (Fig. 4), which are significant functional contents in follicular development such as, membrane traffic protein, receptor, signaling molecule and transcription factor (Additional file 6: Table S5).

\section{Correlation analysis between proteome and transcriptome}

Correlation analysis was carried out by gene name, and proteins without gene name were not involved in the analysis. 15,520 genes (bovine RefSeq database containing 35,325 annotated transcripts) were obtained from the RNA-seq (RPKM cut off $\geq 0.5$ ) (Additional file 7), of which 3978 differentially expressed genes were selected by GEO database (fold change $\geq 2$ ), integrated into proteome data to further identify proteins of consistent expression profile by Venn diagram (Fig. 5). In total, 13 genes overlapped for their mRNAs and proteins expression status and other genes were expressed either in the form of transcript or protein only (Additional file 8).

\section{Changes of proteome profiles}

To compare the expression intensity of 13 differentially expressed proteins in DF and SF, a heatmap was applied (Fig. 6). The heatmap provides visual information of the data, where follicle samples were displayed as columns and classified as indicated by red color and green color, the abbreviations of 13 differentially expressed proteins listed on the right of the heatmap, sequential palettes fix the lowest and the highest value of proteins expression. 
Table 1 Top 30 highly differentially expressed proteins in DF and SF

\begin{tabular}{|c|c|c|c|c|c|}
\hline uniprot & protein name & DF mean & SF mean & DF/SF & $P$ value \\
\hline A5D9E8 & OGN & $23,344,333,333$ & $18,603,000,000$ & 12.034 & 0.041 \\
\hline P01045 & KNG2 & $17,741,666,667$ & $16,726,333,333$ & 6.610 & 0.018 \\
\hline A6QLS5 & MGC143209 & $15,843,333,333$ & $12,345,666,667$ & 5.764 & 0.041 \\
\hline Q3SZZ9 & FGG & $8,715,066,667$ & $9,154,200,000$ & 5.624 & 0.018 \\
\hline F1MAV0 & $\mathrm{FGB}$ & $7,831,366,667$ & $6,321,800,000$ & 5.584 & 0.019 \\
\hline P62739 & ACTA2 & $5,331,333,333$ & $5,513,366,667$ & 5.382 & 0.011 \\
\hline A7YWQ4 & SNTB2 & $4,380,900,000$ & $5,185,966,667$ & 5.239 & 0.036 \\
\hline A5PJE3 & FGA & $2,550,033,333$ & $4,518,066,667$ & 5.053 & 0.007 \\
\hline E1BGS2 & ACAD10 & $2,491,466,667$ & $4,378,000,000$ & 4.894 & 0.001 \\
\hline E1BDU0 & NUP107 & $2,489,033,333$ & $4,180,776,667$ & 4.084 & 0.004 \\
\hline G3X6T9 & FLOT2 & $2,103,033,333$ & $4,086,033,333$ & 3.533 & 0.028 \\
\hline F1 N102 & $\mathrm{C} 8 \mathrm{~B}$ & $1,509,833,333$ & $3,429,200,000$ & 3.495 & 0.041 \\
\hline G3X715 & $4 \mathrm{SV}$ & $1,368,263,333$ & $3,154,466,667$ & 3.398 & 0.012 \\
\hline F6RB08 & KIF17 & $1,288,343,333$ & $3,135,646,667$ & 3.375 & 0.046 \\
\hline F1MTK4 & LPCAT1 & $1,208,773,333$ & $2,667,900,000$ & 2.924 & 0.028 \\
\hline F1NOF2 & SIAE & $1,094,433,333$ & $2,598,433,333$ & 2.878 & 0.001 \\
\hline A8YXZ2 & C8G & $1,036,296,667$ & $2,314,433,333$ & 2.799 & 0.050 \\
\hline G3MZ95 & FHL1 & $966,820,000$ & $2,305,566,667$ & 2.704 & 0.047 \\
\hline A1L5A6 & ADRM1 & $906,270,000$ & $1,791,266,667$ & 2.565 & 0.036 \\
\hline P41976 & SOD2 & $761,470,000$ & $1,637,366,667$ & 2.539 & 0.002 \\
\hline D3K0R6 & ATP2B4 & $750,666,666.7$ & $1,625,866,667$ & 2.427 & 0.004 \\
\hline E1BHR3 & HDGFRP3 & $592,620,000$ & $1,380,866,667$ & 2.373 & 0.024 \\
\hline F1 MJ71 & COL4A4 & $561,006,666.7$ & $1,348,310,000$ & 2.238 & 0.002 \\
\hline Q3ZBT5 & STX7 & $525,786,666.7$ & $1,292,716,667$ & 2.156 & 0.002 \\
\hline E1BMM0 & NCBP1 & $511,866,666.7$ & $1,217,820,000$ & 2.151 & 0.015 \\
\hline F6RQK3 & GSTZ1 & $511,250,000$ & $1,204,496,667$ & 2.116 & 0.009 \\
\hline F1MUX6 & GSTM3 & $509,750,000$ & $1,165,810,000$ & 0.496 & 0.038 \\
\hline E1BHJO & $3 \mathrm{SV}$ & $472,130,000$ & $1,121,570,000$ & 0.494 & 0.011 \\
\hline F1MNG3 & RAC1 & $470,410,000$ & $1,030,903,333$ & 0.485 & 0.031 \\
\hline Q3TOF4 & RPS10 & $468,846,666.7$ & $1,004,480,000$ & 0.484 & 0.026 \\
\hline
\end{tabular}

These results elucidated 8 proteins (RPL17, RPS26, DAZAP1, GUK1, RPS20, OLA1, CCT5, STIP1) highly expression in SF, lowly expression in DF, while 5 proteins (OGN, ACTA2, FGA, ATP2B4, C8B) highly expression in DF, lowly expression in SF.

\section{Immunoblot validation}

The expression tendency of three proteins were consistent with MS data.

\section{Discussion}

This study represents the first investigation of multiomics correlation analysis of bovine follicles and identified numerous new candidate proteins potentially interacting with mechanism of follicular development.
We found that specific highly differentially expressed proteins in DF, including fibrinogen (e.g.,FGA, FGB, and FGG), OGN and ACTA2, etc. FGA, OGN and ACTA2 are also 3 proteins of 13 differential associated proteins. Fibrinogen is an essential component of hemostatic system, normal pregnancy is associated with hypercoagulable state, fibrinogen increased progressively throughout pregnancy [24], it is suggested that fibrinogen might be a critical factor in follicle. OGN and ACTA2 are associated with tumors, and both inhibit cell proliferation. OGN expression in DF is highest compared with SF in 26 upregulated proteins, previous studies have shown loss of OGN expression in cancer cell lines and different tumors [25], suggesting its potential role as a tumor-suppressor gene. ACTA2 has positive effects on metastatic potential, it could be a promising therapeutic target for metastatic 


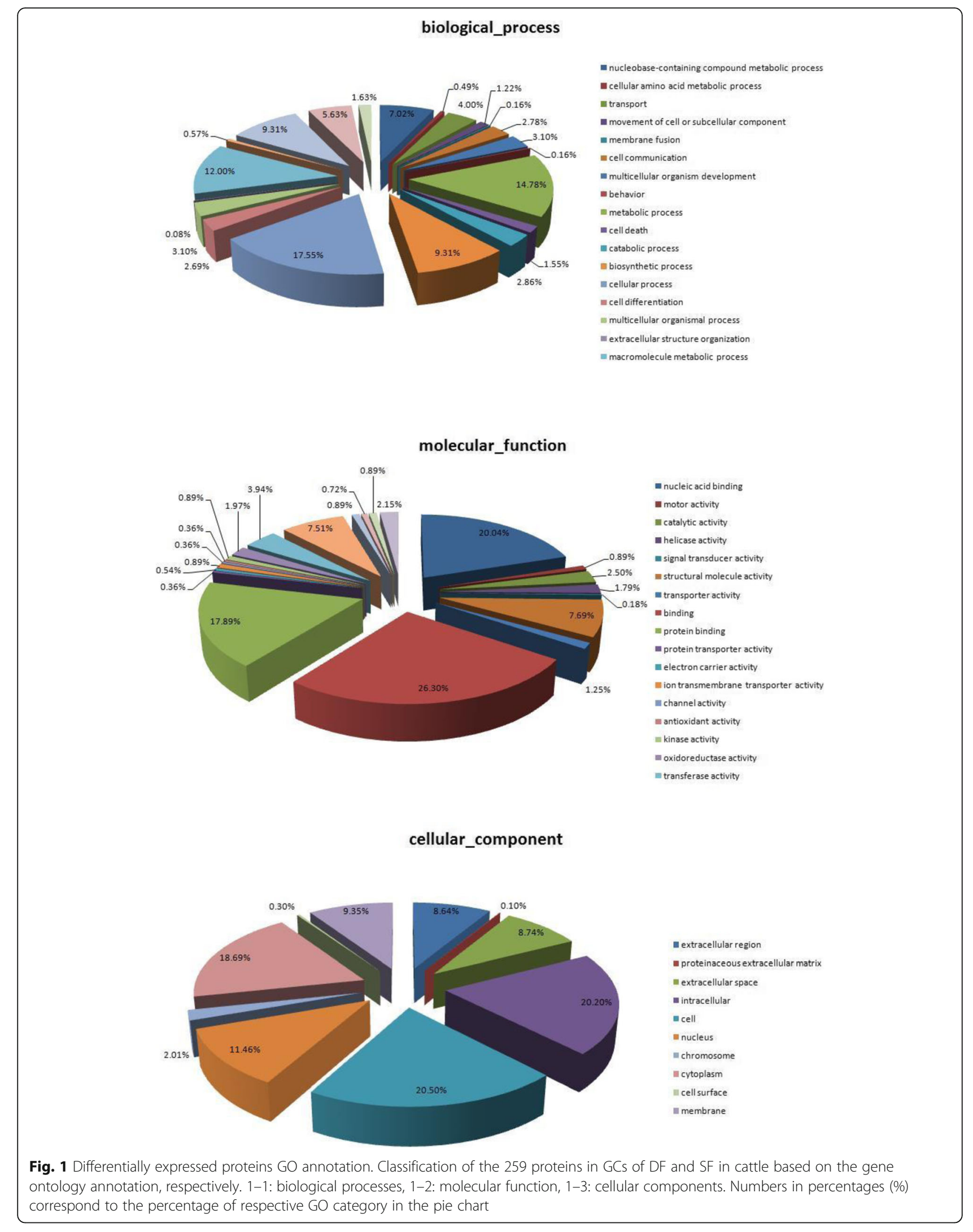




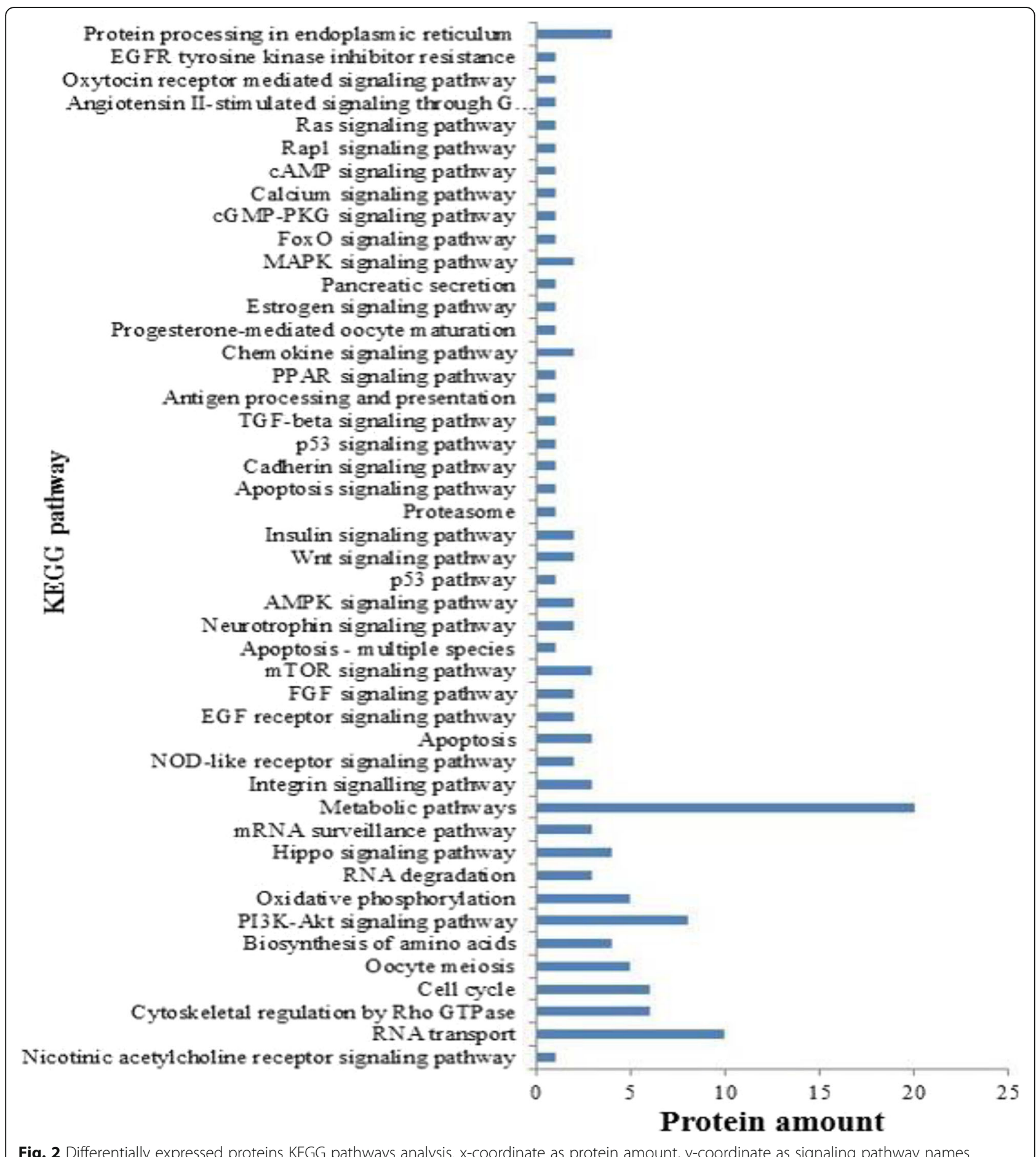

Fig. 2 Differentially expressed proteins KEGG pathways analysis. x-coordinate as protein amount, $y$-coordinate as signaling pathway names

lung adenocarcinoma [26]. Cattle undergoes 2-3 follicular wave during one estrous cycle, only the DF of last follicle wave eventually ovulation. In this work, DF was selected from the first follicle wave, so DF also undergoes atresia. It is likely that OGN and ACTA2 are associated with anovulatory DF.
We also identified specific highly differentially expressed proteins in SF compared with DF, including 14-3-3 proteins (e.g., YWHAG, YWHAH, YWHAB, YWHAQ), ROR2, PA2G4, DAZAP1, OLA1, eukaryotic translation initiation factors (e.g.,EIF2S3, EIF4A1, EIF1, EIF3I, EIF5A, EIF2A, EIF4G2, EIF3J, EIF1B), DDX1 and CCT6A. 14-3-3 


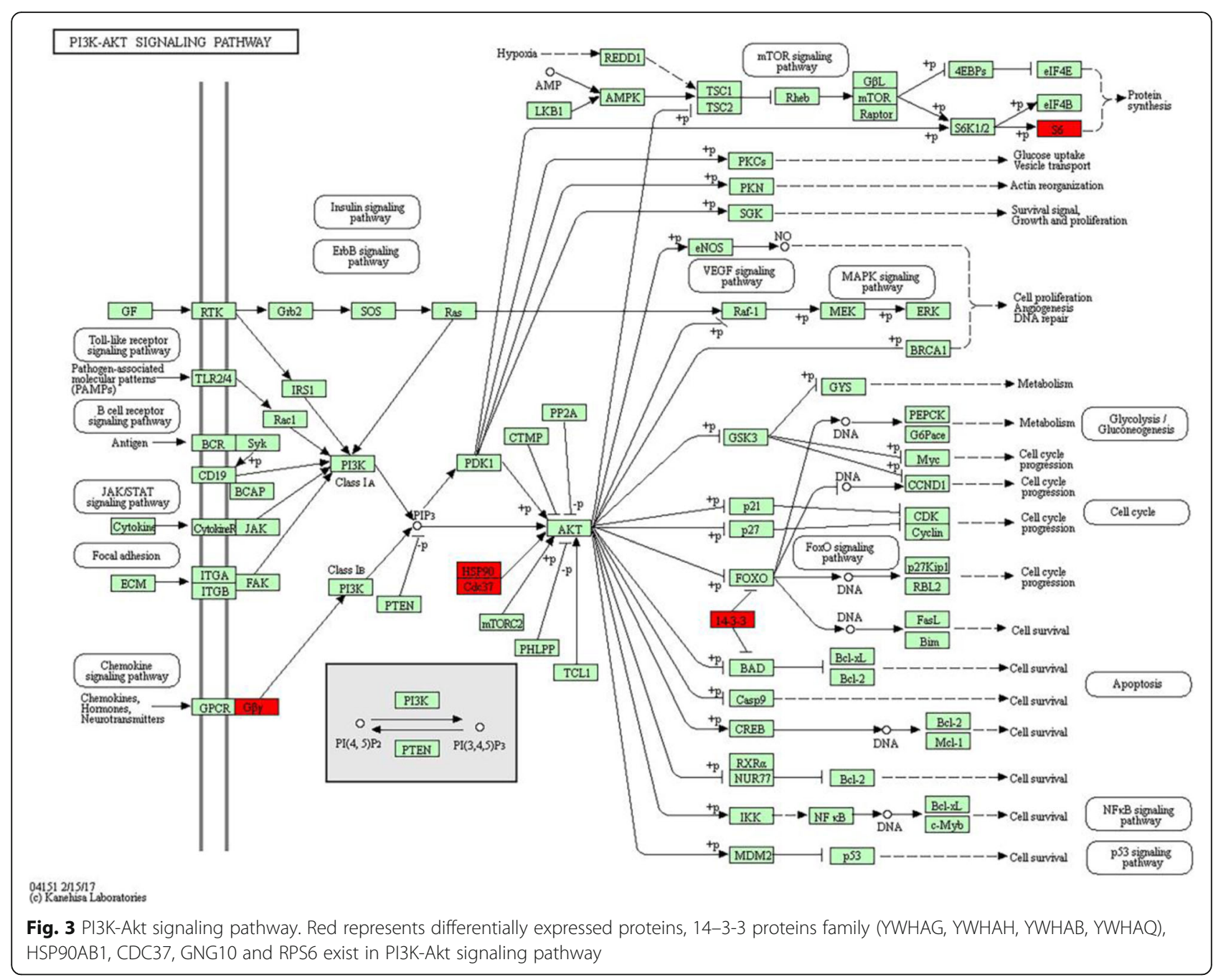

proteins, are considered as anti-apoptotic and critical regulatory proteins in cell division and apoptosis, might also play an important role in regulating cellular activities by associating with cytoskeletal proteins [27]. ROR2 expression is significantly increased in ovarian cancer [28]. PA2G4 is shown to regulate cell differentiation and growth [29]. DAZAP1, a ubiquitously expressed protein, is essential for the normal growth and development of mice [30]. OLA1 is a translational regulator of $\mathrm{p} 21$, which has an important impact on promoting cell proliferation [31]. Accumulatied evidence suggests that eukaryotic initiation involves a large number of eukaryotic initiation factors [32-34], 9 eukaryotic translation initiation factors and ATP-dependent RNA helicase DDX1 were found in proteome data, which are correlated with translation initiation [35] and cell proliferation [36]. It is suggested that14-3-3 protein family (YWHAG, YWHAH, YWHAB, YWHAQ), ROR2, PA2G4, DAZAP1, OLA1, eukaryotic translation initiation factors and DDX1 are likely to be important proteins in cell proliferation. CCT6A likely plays important role in follicle growth and sexual maturity in hens [37]. In GCs isolated from preovulatory follicles, progesterone activated CCT6A, whereas follicle-stimulating hormone (FSH) inhibited the expression of CCT6A mRNA [38]. FSH targets GCs exclusively to induce their differentiation and maturation [39], the onset of each follicular wave is preceded by FSH transient increase, whereas DF selection occurs in the face of declining FSH concentrations [40], so CCT6A expression level is lower in DF. Unequivocally, these downregulated proteins were associated with cell growth. It's well known that SF finally developed into atresia follicle, paradoxically, highly expression of these proteins could lead to GCs proliferation, which needs to be further research.

GO analysis of differentially expressed proteins showed most of the proteins distributed in cytoplasm of the cell, and involved in nucleic acid binding and protein binding, and associated with metabolic process, macromolecule metabolic process, biosynthetic process, regulation of biological process. Therefore, it appears that most of these proteins are involved in regulation of metabolism. 

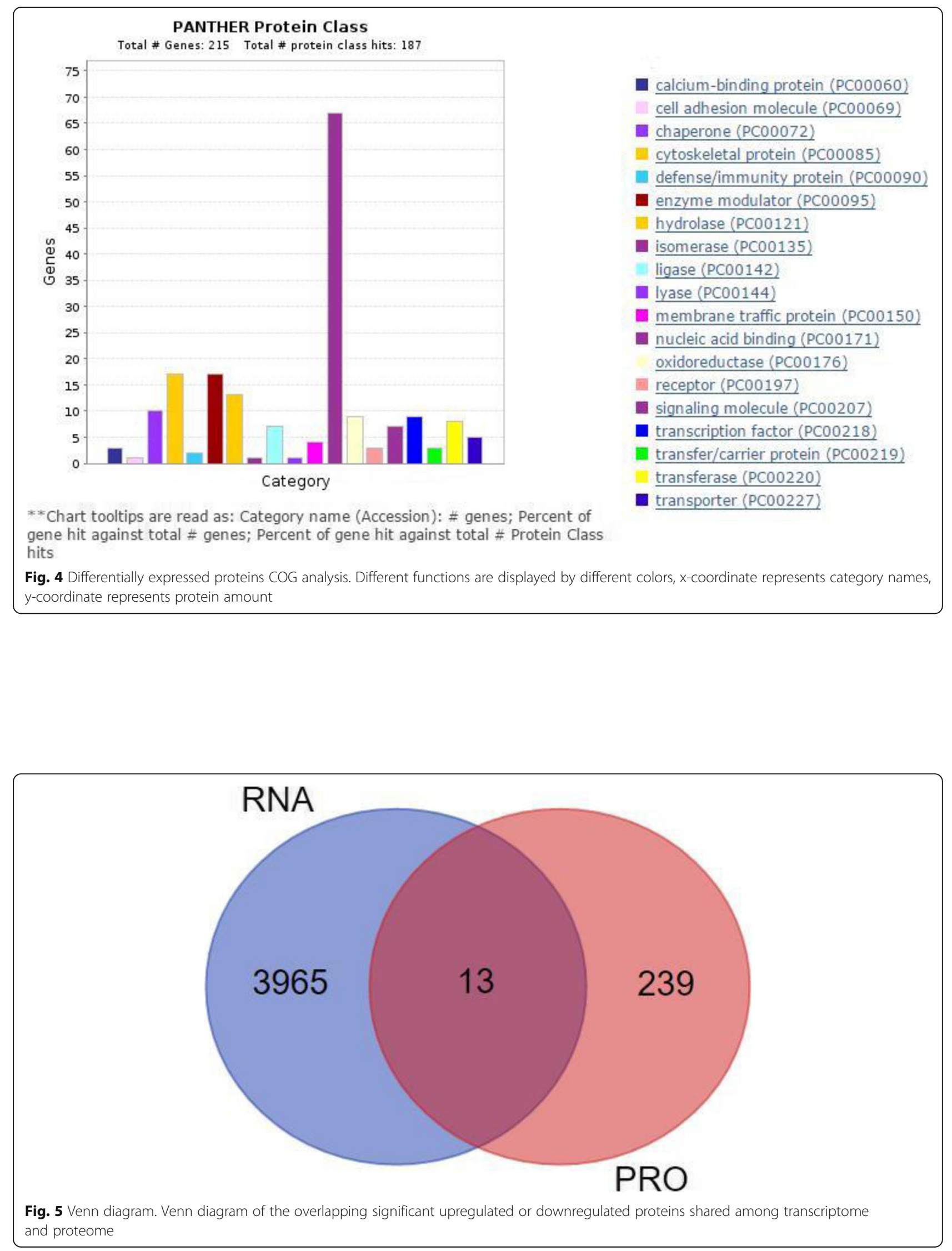


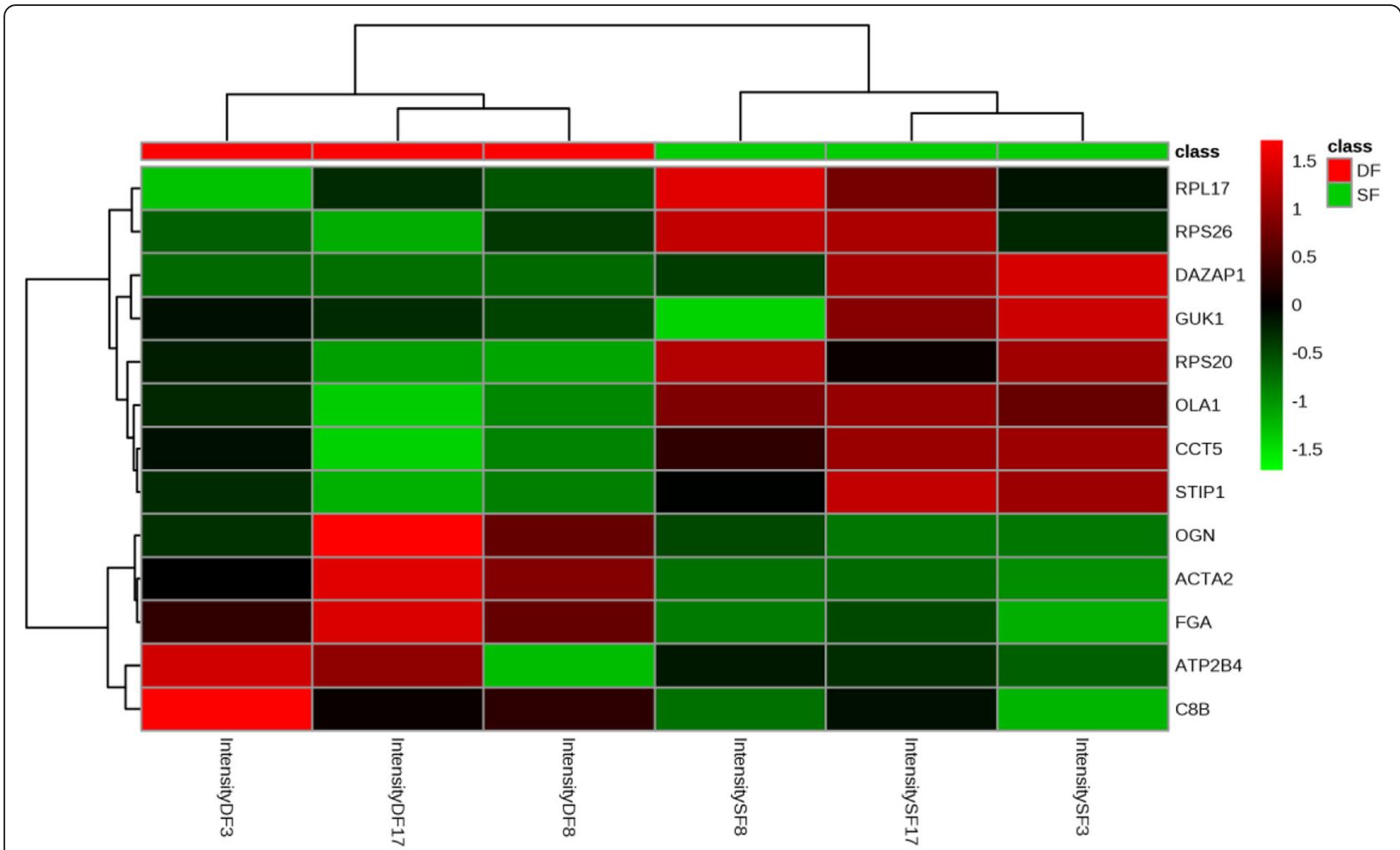

Fig. 6 Heatmap. The heatmap presents relative abundance of the proteins with different colors, where deeper green represents lower intensity and deeper red represents higher intensity. Different Samples are displayed as columns and classified by proteomic subtypes as indicated by different colors. The gene names listed on the right of the heatmap

To clarify the metabolic pathways in which these proteins were involved, we performed KEGG pathway analysis, 46 signaling pathway are listed in Fig. 2, of these are associated with follicular development and possess differentially expressed proteins, such as PI3K-Akt signaling pathway, estrogen signaling pathway, progesterone-mediated oocyte maturation, mTOR signaling pathway, apoptosis, wnt signaling pathway, insulin signaling pathway, metabolic pathways. It is known that Wnt signaling is essential for embryonic development and cellular processes [41], insulin is crucial for GCs function, follicle growth and ovulation in cows [42], PI3K-Akt pathway plays an important role in follicular development, estradiol initiate estrous behavior, which is critical to sustain DF growth [43], etc. This also verify the accuracy of proteome results. Interestingly, 14-33 proteins (e.g., YWHAG, YWHAH, YWHAB, YWHAQ) were not only highly differentially expressed proteins in SF, but enriched in both PI3K-Akt signaling pathway and Hippo signaling pathway. Therefore, YWHAG, YWHAH, YWHAB, YWHAQ may be the primary proteins responsible for follicular development. Oxidative stress may be related to folliculogenesis and oogenesis [44] in the bovine species, SOD2 - mitochondrial can act directly on superoxide anion radicals, especially, estradiol inhibited SOD2
mRNA expression in rat luteal cells [45]. KEGG analysis showed that SOD2 participate in FoxO signaling pathway, and SOD2 was upregulated in DF, this may indicate that SOD2 may play an important role in DF.

In the current study, most genes that differentially expressed in proteome data, whereas not differentially expressed in transcriptome data, so only 13 differentially expressed proteins were identified through omics association analysis. Furthermore, regulation of mRNA turnover in the cell is essential for controlling the abundance of cellular transcripts, in turn, the levels of protein expression [46, 47]. mRNA half-life is linked to the function of the encoded protein, substantial stability of a mRNA means that it will be available for translation for a longer time, leading to high levels of protein gene products. mRNAs of many regulatory genes, which encode proteins that are required for only a short timesuch as regulators of growth or differentiation, cell cycle regulators-often have short half-lives [48], so mRNAs of some proteins might not be detected because of mRNAs degradation, such as ELOC and SERPINA3 are differentially expressed proteins, which RNA were not found in transcriptome data. Recent studies revealed that ribosomal proteins (RPs) have been associated with 
cell proliferation, survival, apoptosis and other cellular processes, such as RPS20 was able to increase cell cycle arrest and cell death, and extend the half-life of p53 by more than 4-fold [49]. 31 RPs are all down-regulated in proteome data, and specifically, the expression of RPS26, RPL17, RPS20 decrease in both transcript and protein levels, It is suggested that RPS26, RPL17, RPS20 possess stable functions and may play an important role in the follicular development.

\section{Conclusions}

The present study characterized the GCs proteome of bovine follicles at specific stages by a label-free strategy, a total of 259 proteins were identified as differentially expressed proteins (fold Change $>2, P<0.05$ ), 13 differentially expressed proteins were identified in DF versus SF by performing translatomics integrated with proteomics analysis, some proteins of 259 differentially expressed proteins may be associated with follicular development, including eukaryotic translation initiation factors (e.g.,EIF2S3, EIF4A1, EIF1, EIF3I, EIF5A, EIF2A, EIF4G2, EIF3J, EIF1B), 14-3-3 proteins (e.g., YWHAG, YWHAH, YWHAB, YWHAQ), fibrinogen (e.g., FGB and FGG), etc. Some proteins whose mRNA are absent because of short mRNA half-life. Bioinformatics analyses also provided metabolic pathway information about these proteins, however, much more research is needed to confirm that these proteins are biomarkers for follicular development.

\section{Supplementary information}

The online version of this article (https://doi.org/10.1186/s12953-019-0152-1) contains supplementary material, which is available to authorized users.

Additional file 1: Table S1. List of proteins (3409) expressed in DF and SF from the 30,321 peptides (FDR $\leq 0.01)$.

Additional file 2: Table S2. List of genes $(15,520)$ expressed in DF and SF follicles with a cut-off RPKM of 0.5. Table S2. List of 259 differentially expressed proteins in DF and SF (Fold Change $>2, P<0.05$ ).

Additional file 3: Table S3. List of Go analysis of 259 differentially expressed proteins.

Additional file 4: Table S4. List of KEGG pathway analysis of 259 differentially expressed proteins.

Additional file 5. Eight differentially expressed proteins in PI3K-Akt signaling pathway.

Additional file 6: Table S5. List of COG analysis of 259 differentially expressed proteins.

Additional file 7: Table S6. List of genes $(15,520)$ expressed in DF and SF follicles with a cut-off RPKM of 0.5 .

Additional file 8: Table S7. List of 13 differentially expressed genes in DF and SF.

\section{Abbreviations}

AGC: Automatic gain control; CID: Collision-induced dissociation; COG: Cluster of Orthologous Groups; DF: Dominant follicle; FDR: False discovery rate; FSH: Follicle-stimulating hormone; GCs: Granulosa cells; GO: Gene ontology; IGF-1: Insulin-like growth factors 1; LC-MS/MS: Label-free liquid chromatography-tandem mass spectrometry; PGF2a: Prostaglandin
F2a; RPs: Ribosomal proteins; SF: Subordinate follicle; VEGF: Vascular endothelial growth factor

\section{Acknowledgements}

Authors are grateful of Prof. Sandeep Kumar Rajput and Prof. James Richard Pursley in Michigan State University in the USA for their directions of research designs and manuscript preparation.

\section{Authors' contributions}

HS and LP conceived the idea of this study. LW and LL collected samples. $H S, H Q, Z Z$, and XD performed lab works. HS and LP wrote the manuscript. All authors read and approved the final manuscript.

\section{Funding}

This study was supported by Shanxi Scholarship Council of China Grant no. 2014-key 5, Shanxi Sci-technological Collaboration Grant no. 201603D421006, Shanxi Talent Introduction and Sanjin Talent Program, Shanxi Provincial Talent Introduction and SXAU (Shanxi Agricultural University) Major Research Achievement Cultivation Grant no. zdpy 201403/201503 to Lyu; Chinese Natural Science Foundation Grant no. 31873002, Shanxi Key Research and Development Plan (general) Agriculture Project Grant no. 201703d221020-1 to Li.

\section{Availability of data and materials}

Availability of data and materials are included in the manuscript, figures and tables.

Ethics approval and consent to participate

We confirm that this study did not involve relevant clause of the Ethics Committee, and all animal procedures were performed in strict accordance with the recommendations in the Guide for the Care and Use of Laboratory Animals of the National Institutes of Health.

\section{Consent for publication}

All authors read and approved the final manuscript, and consented for publication

\section{Competing interests}

The authors declare that they have no competing interests.

Received: 3 April 2019 Accepted: 16 September 2019

Published online: 25 October 2019

\section{References}

1. Fortune JE, Rivera GM, Evans AC, et al. Differentiation of dominant versus subordinate follicles in cattle. Biol Reprod. 2001;65(3):648-54.

2. Diskin MG, Mackey DR, Roche JF, et al. Effects of nutrition and metabolic status on circulating hormones and ovarian follicle development in cattle. Anim Reprod Sci. 2003;78(3-4):345-70.

3. Langbeen A, De Porte HF, Bartholomeus E, et al. Bovine in vitro reproduction models can contribute to the development of (female) fertility preservation strategies. Theriogenology. 2015;84(4):477-89.

4. Roche JF, Austin EJ, Ryan M, et al. Regulation of follicle waves to maximize fertility in cattle. J Reprod Fertil Suppl. 1999;54:61-71.

5. Ginther OJ, Wiltbank MC, Fricke PM, et al. Selection of the dominant follicle in cattle. Biol Reprod. 1996;55(6):1187-94.

6. Webb R, Armstrong DG. Control of ovarian function; effect of local interactions and environmental influences on follicular turnover in cattle: a review. Livest Prod Sci. 1998:53(2):95-112.

7. Wei XH, Qi LH, Chi XC, et al. The effects of FSH on the phosphorylation of smad2/smad3 protein in rat ovarian granulosa cells. Acta Anatomica Sinica. 2007;38(2):205-8.

8. Pedersen $\mathrm{HG}$, Watson ED, Telfer EE. Analysis of atresia in equine follicles using histology, fresh granulosa cell morphology and detection of DNA fragmentation. Reproduction. 2003;125(3):417-23.

9. Zeuner A, Müller K, Reguszynski K, et al. Apoptosis within bovine follicular cells and its effect on oocyte development during in vitro maturation. Theriogenology. 2003;59(5-6):1421-33.

10. Wongsrikeao P, Kaneshige $Y$, Ooki R, et al. Effect of the removal of cumulus cells on the nuclear maturation, fertilization and development of porcine oocytes. Reprod Domest Anim. 2005;40(2):166-70. 
11. Dias FC, Khan Ml, Adams GP, et al. Granulosa cell function and oocyte competence: super-follicles, super-moms and super-stimulation in cattle. Anim Reprod Sci. 2014;149(1-2):80-9.

12. Kafi M, Mesbah SF, Davoodian N, et al. Fine structures of the oocyte in relation to serum, follicular fluid steroid hormones and IGF-I in the ovulatory-sized follicles in one-humped camel (Camelus dromedarius). Avicenna J Med Biotechnol. 2014;6(1):57-61.

13. Aad PY, Echternkamp SE, Spicer $\sqcup$. Possible role of IGF2 receptors in regulating selection of 2 dominant follicles in cattle selected for twin ovulations and births. Domest Anim Endocrinol. 2013;45(4):187-95.

14. Babitha V, Panda RP, Yadav VP, et al. Amount of mRNA and localization of vascular endothelial growth factor and its receptors in the ovarian follicle during estrous cycle of water buffalo (Bubalus bubalis). Anim Reprod Sci. 2013;137(3-4):163-76.

15. Gupta S, Choi A, Yu HY, et al. Fluctuations in total antioxidant capacity, catalase activity and hydrogen peroxide levels of follicular fluid during bovine folliculogenesis. Reprod Fertil Dev. 2011;23(5):673-80.

16. Li P, Meng J, Liu W, et al. Transcriptome analysis of bovine ovarian follicles at Predeviation and onset of deviation stages of a follicular wave. Int J Genomics. 2016;2016:1-9.

17. Lindsey ML. PROTEOMICS: the journey ahead... from the principles of life to the development of new therapies. Proteomics. 2016;16(1):4-5.

18. Shalit T, Elinger D, Savidor A, et al. MS1-based label-free proteomics using a quadrupole orbitrap mass spectrometer. J Proteome Res. 2015;14(4):1979-86.

19. Thermo Scientific. Xcalibur proteome discoverer version 1.3 user guide. 2011, 5.

20. Matrix Science. Introduction to database searching using MASCOT. Mascot 2.5.

21. Li S, Tang H. Computational methods in mass spectrometry-based proteomics. Adv Exp Med Biol. 2016;939:63-89.

22. Sandberg A, Lindell G, Källström BN, et al. Tumor proteomics by multivariate analysis on individual pathway data for characterization of vulvar cancer phenotypes. Mol Cell Proteomics. 2012;11(7):M112.016998.

23. Hao QL, Zhu ZW, Xu DM, et al. Proteomic characterization of bovine granulosa cells in dominant and subordinate follicles. Hereditas. 2019;156:21.

24. Réger B, Péterfalvi A, Litter I, et al. Challenges in the evaluation of D-dimer and fibrinogen levels in pregnant women. Thromb Res. 2013;131(4):e183-7.

25. Tasheva ES, Maki CG, Conrad AH, et al. Transcriptional activation of bovine mimecan by 553 through an intronic DNA-binding site. Biochim Biophys Acta. 2001;1517(3):333-8

26. Lee HW, Park YM, Lee SJ, et al. Alpha-smooth muscle actin (ACTA2) is required for metastatic potential of human lung adenocarcinoma. Clin Cancer Res. 2013;19(21):5879-89.

27. van Hemert MJ, Steensma HY, van Heusden G. P. 14-3-3 proteins: key regulators of cell division, signalling and apoptosis. Bioessays. 2001;23(10):936-46.

28. Claire H, Estelle L, Alexandra KM, et al. Targeting the ROR1 and ROR2 receptors in epithelial ovarian cancer inhibits cell migration and invasion. Oncotarget. 2015;6(37):40310-26.

29. Xia X, Cheng A, Lessor T, et al. Ebp1, an ErbB-3 binding protein, interacts with $\mathrm{Rb}$ and affects $\mathrm{Rb}$ transcriptional regulation. J Cell Physiol. 2001;187(2): 209-17

30. Hsu CL, Chen HY, Lin YW, et al. DAZAP1, an hnRNP protein, is required for normal growth and spermatogenesis in mice. RNA. 2008;14(9):1814-22.

31. Ding Z, Liu Y, Rubio V, et al. OLA1, a translational regulator of p21, maintains optimal cell proliferation necessary for developmental progression. Mol Cell Biol. 2016;36(20):2568-82.

32. Hershey JW. Translational control in mammalian cells. Annu Rev Biochem. 1991;60:717-55.

33. Merrick WC. Mechanism and regulation of eukaryotic protein synthesis. Microbiol Rev. 1992;56(2):291-315

34. Pain VM. Initiation of protein synthesis in eukaryotic cells. Eur J Biochem. 1996;236(3):747-71.

35. Taunk NK, Goyal S, Wu H, et al. DEAD box 1 (DDX1) expression predicts for local control and overall survival in early stage, node-negative breast cancer. Cancer. 2012;118(4):888-98

36. Caraglia M, Marra M, Giuberti G, et al. The role of eukaryotic initiation factor $5 \mathrm{~A}$ in the control of cell proliferation and apoptosis. Amino Acids. 2001; 20(2):91-104.

37. Kang $L$, Zhang $Y$, Zhang $N$, et al. Identification of differentially expressed genes in ovaries of chicken attaining sexual maturity at different ages. Mol Biol Rep. 2012;39(3):3037-45.

38. Wei Q, Zhu G, Cui X, et al. Expression of CCT6A mRNA in chicken granulosa cells is regulated by progesterone. Gen Comp Endocrinol. 2013;189:15-23.
39. Kumar TR, Wang Y, Lu N, et al. Follicle stimulating hormone is required for ovarian follicle maturation but not male fertility. Nat Genet. 1997;15(2):201-4.

40. Evans A, Canty M J. Physiology of follicle development in cattle. 2004.

41. Chien AJ, Conrad WH, Moon RT. A Wnt survival guide: from flies to human disease. J Invest Dermatol. 2009;129(7):1614-27.

42. Bossaert P, Cock HD, Leroy JLMR, et al. Immunohistochemical visualization of insulin receptors in formalin-fixed bovine ovaries post mortem and in granulosa cells collected in vivo. Theriogenology. 2010;73(9):1210-9.

43. Lunenfeld B, Insler V. Follicular development and its control. Gynecol Endocrinol. 1993;7(4):285-91.

44. Combelles $C$, Holick E, Paolella L, et al. Profiling of SOD isoenzymes in compartments of the developing bovine antral follicle. Reproduction. 2010; 139(5):871-81.

45. Sugino N, Hirosawatakamori M, Zhong L, et al. Hormonal regulation of copper-zinc superoxide dismutase and manganese superoxide dismutase messenger ribonucleic acid in the rat corpus luteum: induction by prolactin and placental lactogens. Biol Reprod. 1998;59(3):599-605.

46. Parker $\mathrm{R}$, Song $\mathrm{H}$. The enzymes and control of eukaryotic mRNA turnover. Nat Struct Mol Biol. 2004;11(2):121-7.

47. Wilusz CJ, Wormington M, Peltz SW. The cap-to-tail guide to mRNA turnover. Nat Rev Mol Cell Biol. 2001;2(4):237-46.

48. Chen CYA, Ezzeddine N, Shyu AB, Messenger RNA. Half-life measurements in mammalian cells. Methods Enzymol. 2008;448:335-57.

49. Daftuar L, Zhu Y, Jaca X, Prives C. Ribosomal proteins RPL37, RPS15 and RPS20 regulate the Mdm2-p53-MdmX network. PLoS One. 2013;8(7):e68667.

\section{Publisher's Note}

Springer Nature remains neutral with regard to jurisdictional claims in published maps and institutional affiliations.
Ready to submit your research? Choose BMC and benefit from:

- fast, convenient online submission

- thorough peer review by experienced researchers in your field

- rapid publication on acceptance

- support for research data, including large and complex data types

- gold Open Access which fosters wider collaboration and increased citations

- maximum visibility for your research: over $100 \mathrm{M}$ website views per year

At BMC, research is always in progress.

Learn more biomedcentral.com/submissions 